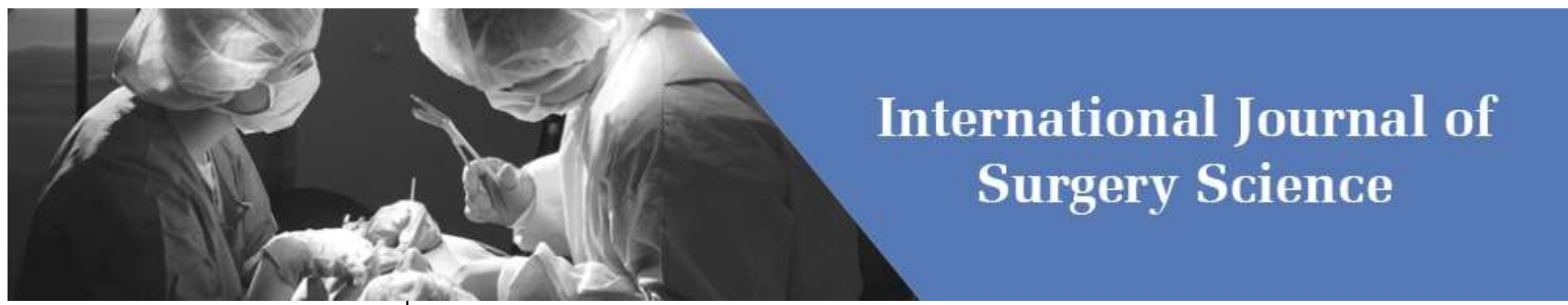

E-ISSN: 2616-3470

P-ISSN: 2616-3462

(c) Surgery Science

www.surgeryscience.com

2021; 5(4): 300-303

Received: 15-08-2021

Accepted: 20-09-2021

Dr. Prakash Modha

Honorary Professor of

Neurosurgery, Pandit Deendayal

Upadhyay Government Medical

College and Chairman Gokul Group

of Hospitals, Rajkot, Gujarat,

India

\section{Micro lumbar discectomy: Review and case report}

\author{
Dr. Prakash Modha
}

DOI: https://doi.org/10.33545/surgery.2021.v5.i4e.801

\section{Abstract}

Lumbar discectomy is the most common elective surgery for degenerative lumbar spine lesions. The rates of good and excellent outcomes are as high as $90-95 \%$. Intervertebral disc prolapse occurs because of the tear in the annulus fibrosis and through which jelly like center portion called nucleus pulposis comes out and causing pressure over the nerve roots. Low-back pain causes nearly $80 \%$ of injured workers to miss at least 8 weeks of work following a back injury. In persons younger than 45 years old, low-back pain is the most frequent cause of activity limitation. Apart from nonspecific back pain, degenerative disease and disc herniation represent the most common causes for LBP-related physician office consultations. In fact, the majority of lumbar radicular pain symptoms are the result of a disc herniation, defined as bulging of the nucleus pulposus through a fissure or tear within the annulus fibrosus. Although favorable outcomes have been demonstrated for both surgical and nonoperative treatment options, patients who underwent open discectomy for lumbar disc herniation (LDH) were shown to have better self-reported outcomes than conservatively treated individuals.

In the present case procedure is done under general anesthesia in prone position on a Bolster or under Wilson's frame with a flat table. Skin incision was made than a lumbo-sacral fascia was cut with the help of electro cautery. Under the microscope paraspinal muscle was stripped off lamina with the help of periosteal dissector (Cobb's dissector) upper $2 / 3^{\text {rd }}$ of the lamina and medial part of the facet joint cut with bone scalpel. Sharp cutting incision is seen in lamina and medial facet. Square shaped incision was made in the disc with the help of No. 15 blade and disc is removed in piece meal or as a big piece if sequestrated. Haemostasis was achieved and patties were removed. Patient is made seat and walk after 6 hours of surgery. Patient is discharged on the next day morning with follow up after 7 days with oral medicines. Micro lumbar discectomy is commonly used very safe procedure, providing direct 3D vision and very effective in relieving the symptoms (98\%) of the patient. It minimize the hospital stay and hence reducing the cost of hospitalization and patient can go on his/her duty earlier.

Keywords: Intervertebral disc prolapsed, lumbar discectomy, lumbo-sacral fascia, scalpel

\section{Introduction}

Lumbar discectomy is the most common elective surgery for degenerative lumbar spine lesions. The rates of good and excellent outcomes are as high as 90-95\%. Micro lumbar discectomy is a very hot topic today in neuro-surgery. Lumbar disc surgery is challenged spine surgeons since the $1^{\text {st }}$ reported case in 1929 by Dandy [1]. In 1934 Mixter \& Barr ${ }^{[2]}$; they have first published the article about removing the herniated lumbar disc trans-durally. In 1938 Pool ${ }^{[3]}$ started using myeloscope for visualization of the nerve roots. In 1939 it was Prof. Love ${ }^{[4]}$ started retracting the dura and removing the disc by inter laminar approach.

Microscope was developed in 1957, Theodore Kurze ${ }^{[5]}$ become the first neuro surgeon to use operating microscope to operate on $8^{\text {th }}$ cranial nerve. Greenwood introduce the bipolar forceps in 1930 and this was further modified by Malli's ${ }^{[6]}$ in 1953 in USA. In 1973 Williams Scoville ${ }^{\text {[7] }}$ developed the technique of radical removal of the disc and decompression of the nerve root anteriorly. He stressed the early mobilization and exercise after surgery. Casper in 1974 from Germany; developed the technique of micro lumber discectomy. In 1977 Yasergil from Swizerland, Casper from Germany and Williams (1978) described the technique of Micro Lumbar Discectomy in detail, which is being practiced till today and it is the gold standard surgery for prolapsed intervertebral disc ${ }^{[8-10]}$.

\section{Causes of prolapsed intervertebral disc}

Intervertebral disc prolapse occurs because of the tear in the annulus fibrosis and through which jelly like center portion called nucleus pulposis comes out and causing pressure over the nerve roots.

\section{Dr. Prakash Modha}

Honorary Professor of

Neurosurgery: Pandit Deendayal Upadhyay Government Medical

College and Chairman Gokul Group of Hospitals, Rajkot, Gujarat,

India 
1. Lifting heavy weights.

2. Obesity.

3. Degenerative conditions.

4. Trauma

Micro lumbar discectomy, procedure of choice for

1. PIVD.

2. Lateral recess stenosis.

3. Excision of osteophytes.

\section{Advantages of micro lumbar discectomies are}

1. 3D vision with bright illumination and magnification.

2. Meticulous search for disc fragment.

3. Small incision of $18 \mathrm{~mm}$ length.

4. Minimal muscle retraction.

5. Minimal blood loss $(20 \mathrm{ml})$.

6. Least morbidity.

7. No urinary catheter required.

8. Patient will walk pain free after 6 hours of surgery (Fig $9 \& 10$ ).

9. Patient can go home next day morning.

10. Can go for job earlier.

\section{Surgical technique}
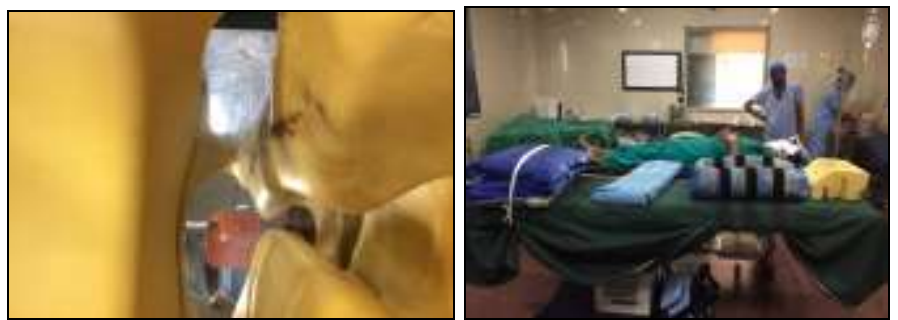

Fig 1: Eye protecting device \& bolster with Velcro

The procedure is done under general anesthesia in prone position on a Bolster or under Wilson"s frame with a flat table. Eyes should be protected with Gamzee and the head should be put in a special device (Fig. 1) so that it should not damage the eyes. It is extremely important that the patient is positioned such that the abdomen hangs free. Pressure on the abdomen will increase pressure within the epidural venous plexus and may lead to annoying and unwanted bleeding during the procedure. The pressure points must be taken care with appropriate sponge. Injection cefuroxime $1.5 \mathrm{gm}$ given before the induction of anesthesia.

A small 18-20 mm long, midline / $1 \mathrm{~cm}$ away from the midline incision (Fig.2) marked at the level of prolapse intervertebral disc with the help of image intensifier. Following preparation, draping and infiltration of the incision marked with a mixture of xylocaine with adrenaline if not contraindicate in the proportion of 50:50 of 1\% xylocaine and 1:2,00,000 adrenaline.
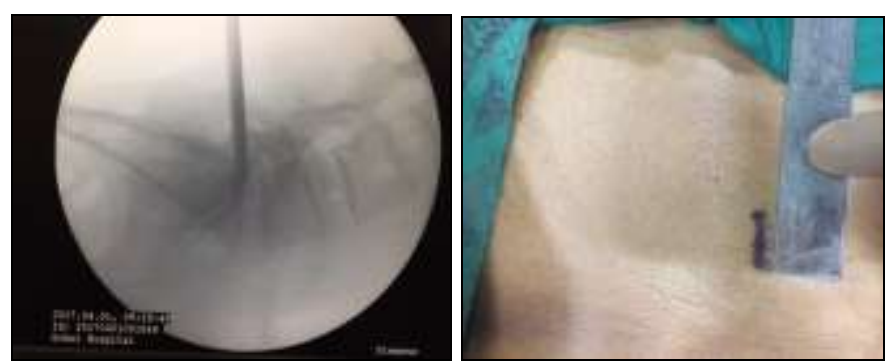

Fig 2: II TV guided marking of skin incision

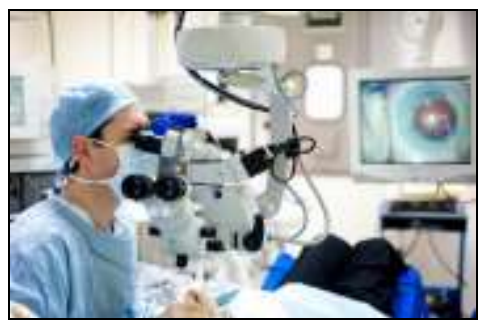

Fig 2: Operating microscope

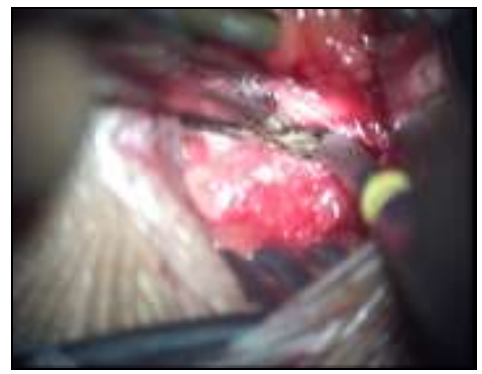

Fig 3: Cutting the lumbo-sacral fascia

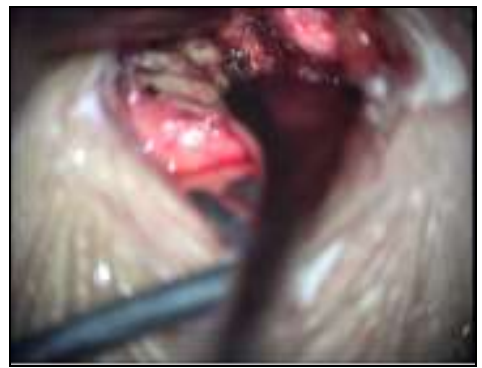

Fig 4: Stripping off the paraspinal muscles from lamina

Skin incision was made than a lumbo-sacral fascia was cut with the help of electro cautery (Fig. 3). Under the microscope paraspinal muscle was stripped off lamina with the help of periosteal dissector (Cobb's dissector) (Fig. 4) then Prof. Ramani's Micro retractors ${ }^{[11]}$ (Fig. 5) were use to retract the muscles, keeping the spike on medial side and blade on lateral side. In case of bilateral micro lumbar discectomy we use blades on both sides.
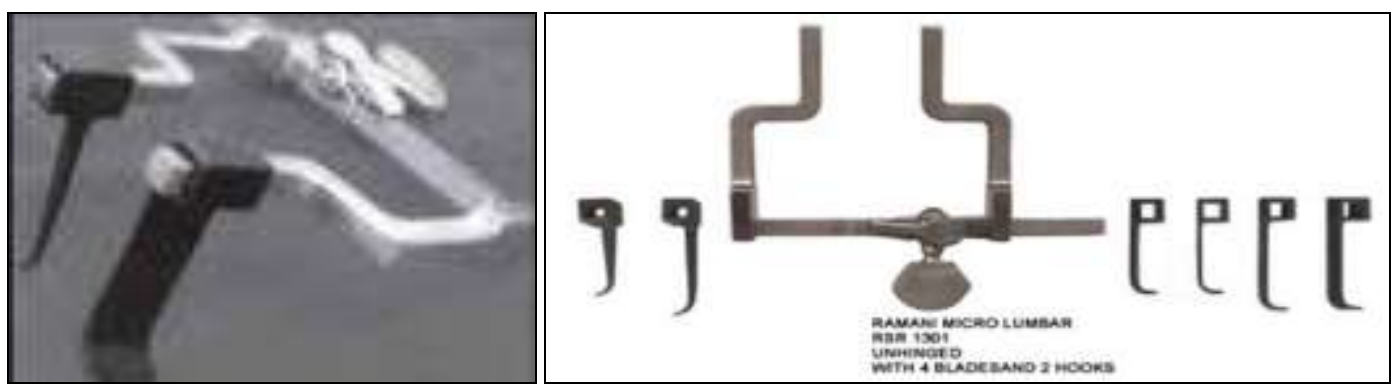

Fig 5: Prof. Ramani's retractor with various blades $\&$ hooks. 


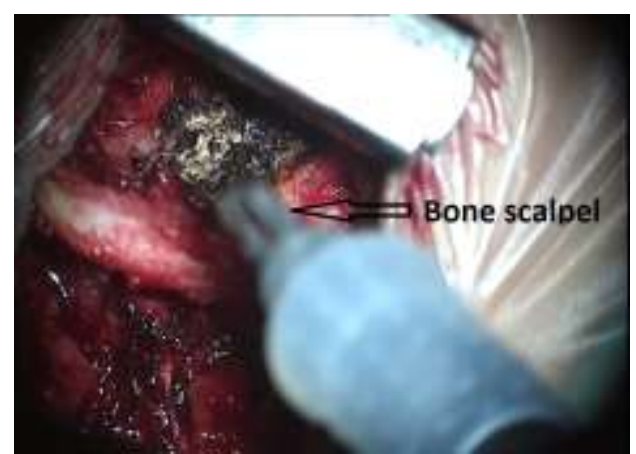

Fig 6: Cutting lamina with bone scalpel

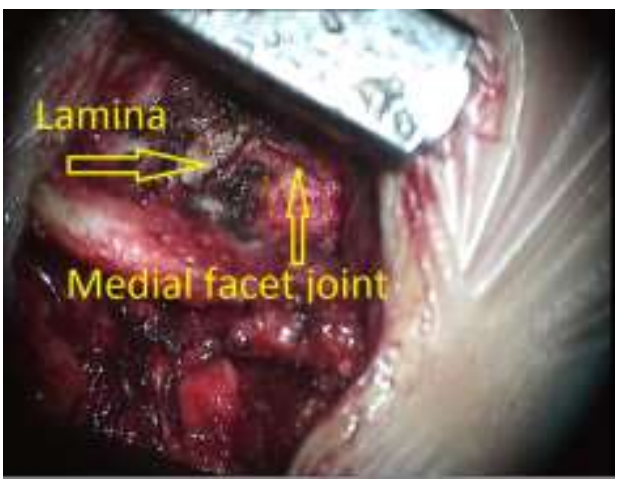

Fig 7: Sharp cut of bone scalpel

Than upper $2 / 3^{\text {rd }}$ of the lamina and medial part of the facet joint cut with bone scalpel (Fig. 6). Sharp cutting incision is seen in lamina and medial facet (Fig. 7). Ligamentumflavum separated from the under surface of the lamina with the help of dura dissector/small curetteand removed. The nerve root is identified under magnification and retracted medially with the help of patties, one above theaxilla of the root and the other at the foramen level. Prolapsed intervertebral disc is identified and tissue over the disc was cauterized with the help of bipolar forceps. Square shaped incision was made in the disc with the help of No. 15 blade and disc is removed in piece meal or as a big piece if sequestrated. Haemostasis was achieved and patties were removed. Abgel was put over the nerve root and depomedrol (Methyle prednisolone) $1 \mathrm{ml}$ (40 mg) was sprinkled. The self retaining retractor was removed.

\section{The closure (Fig. 8):}

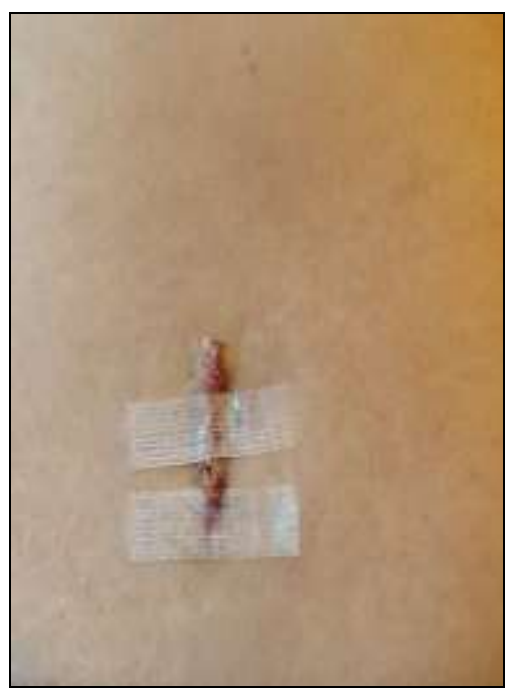

Fig 8: wound closure

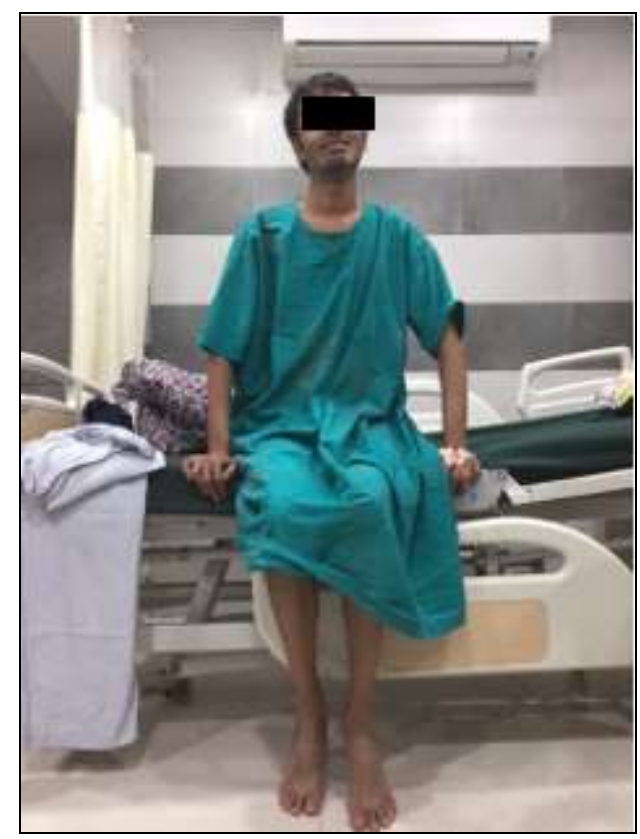

Fig 9: Sitting

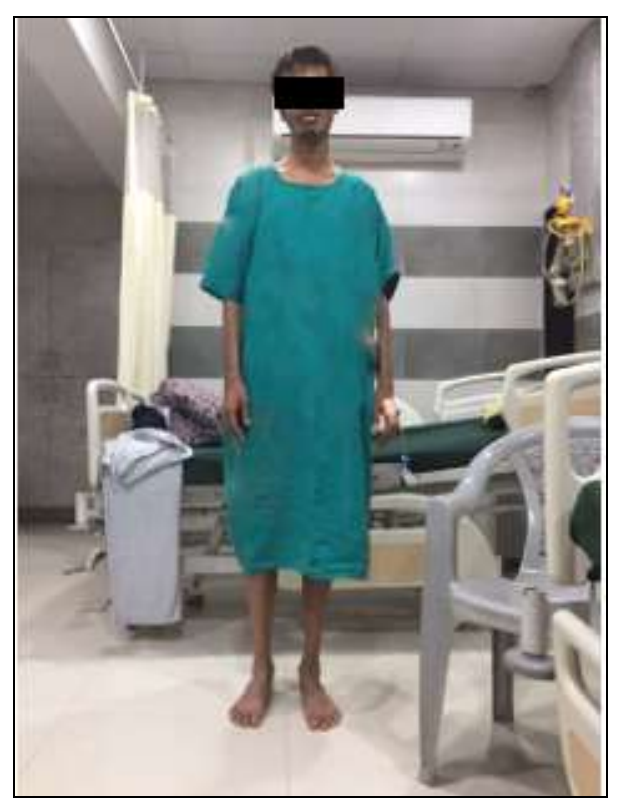

Fig 10: Walking

- No suction drain (Romovac) was kept.

- We do not suture the muscle.

- The lumbo-sacral fascia and the subcutaneous tissue were sutured with 2.0 vicryl (Braided coated polyglactin 910 violet).

- We takes subcuticular stitches with 3.0 monocryl (Monofilament Poliglecaprone 25 undyed).

- A small dressing was kept or we put band-aid stripe.

The surgical procedure can be done in two ways

1. Muscles stripping surgery (Prof. Ramani's micro lumbar discectomy) as described above.

2. Muscles splitting surgery (using medtronics expandable tube retractors or tubular retractors). $1.5 \mathrm{~cm}$ away from the midline paraspinal incision was taken. After cutting skin subcutaneous tissue and fascia, muscles were split by using gradual dilators ${ }^{[12]}$ (Fig. 11). Then expandable tube was inserted and fix with the fixators arm (Fig.12). Rest of the procedure remains the same as open micro discectomy. 


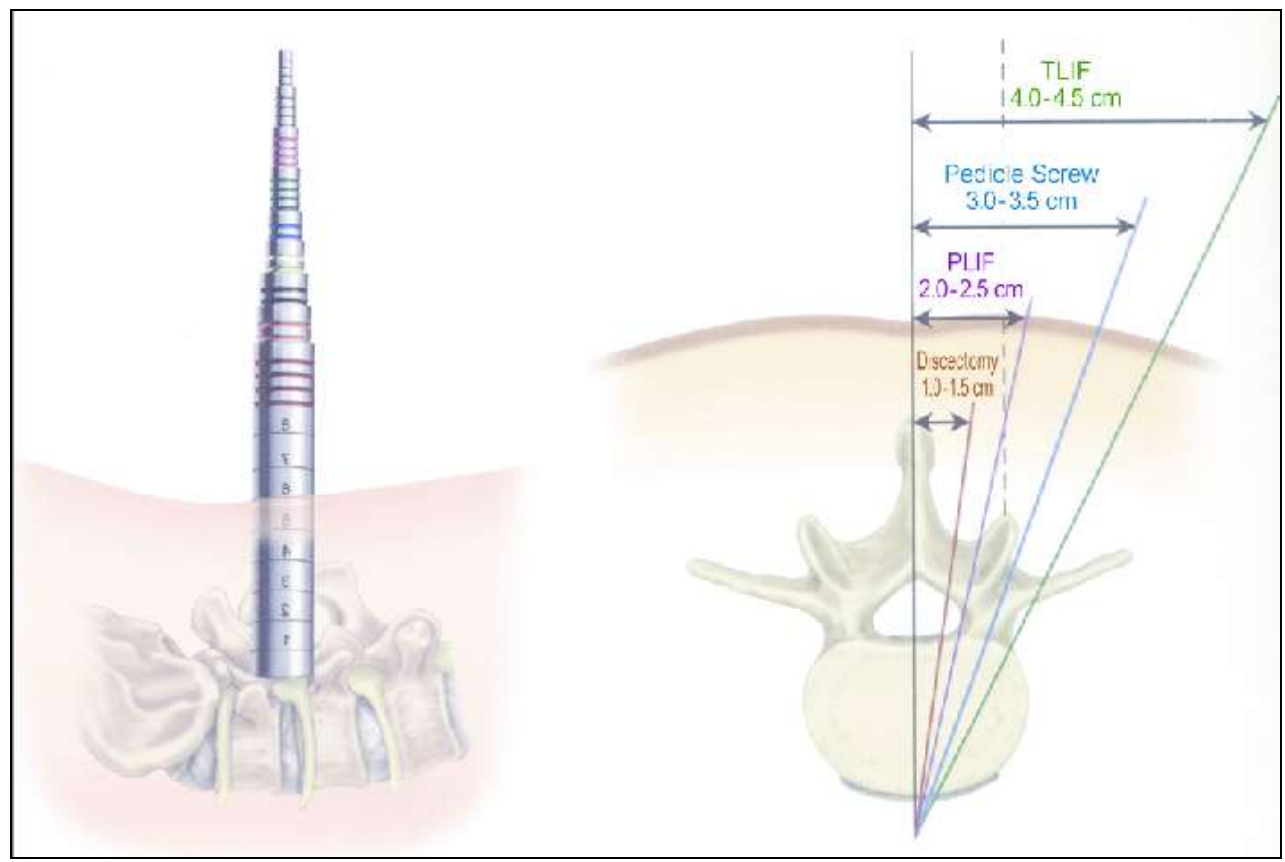

Fig 11: Gradual dilators

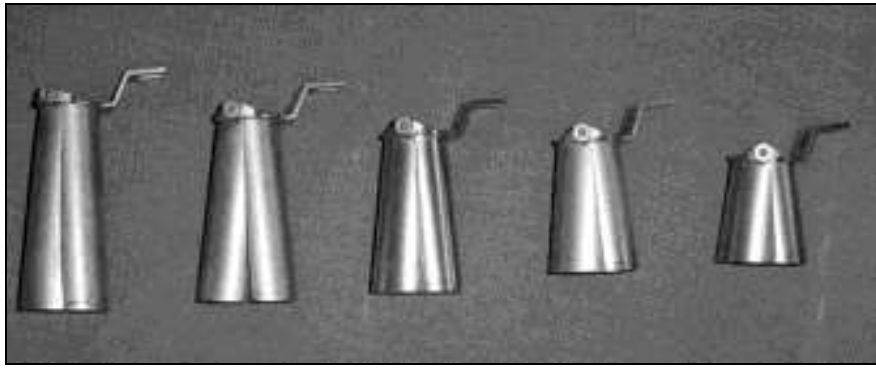

Fig 12: Expandable tubes

\section{Post operative course}

Inj. Cefuroxime sodium $1.5 \mathrm{gm}$ given in the evening and next day morning along with analgesics, antacids and multivitamins. Patient is made seat and walk after 6 hours of surgery (Fig. $9 \&$ 10).

Patient is discharged on the next day morning with follow up after 7 days with oral medicines.

\section{Complications}

1. Infection, very rarely - developed a discities or superficial skin infection. We use injection Carbapenem (Merupenem), Clindamycine (Dalacin C) \&Metronidazole (Metrogyl).

2. Dural tear/CSF leak, rarely encountered. If there is CSF leak or dural tear then it must be repaired immediately by taking suture with 5.0 ethilon and muscle/fat graft must be kept and we should put sealing agent like reliseal (Reliance) or Tisseel fibrin glue (Baxter) or Eviseal (J\&J).

3. Iatrogenic damaging nerve root and pars-interarticularis.

4. Stiffness of the back - which improves with sustained back exercise.

5. List of the spine - improves with exercises in six week of time.

\section{Conclusions}

Micro lumbar discectomy is commonly used very safe procedure, providing direct 3D vision and very effective in relieving the symptoms (98\%) of the patient. It minimize the hospital stay and hence reducing the cost of hospitalization and patient can go on his/her duty earlier.

\section{References}

1. Dandy WE. Loose cartilage from intervertebral disc simulating tumor of the spinal cord. Arch Surg 1929;19:660-672, (Reference unverified).

2. Mister WJ, Barr JS. Rupture of the intervertebral disc with involvement of the spinal canal. $\mathrm{N}$ Engl $\mathrm{J}$ Med 1934;211:210-215,

3. Pool JL. Direct visualization of dorsal nerve roots of the cauda equina by means of a myeloscope," Archives of Neurology and Psychiatry. 1938;39:1308-1312.

4. Love J. Removal of protruded intervertebral disc without laminectomy. Proc Staff Meet Mayo Clain. 1939;14:800805.

5. Ramandeep Singh. A Project Report on - Operating microscope in neuro-surgery, Center for Biomedical Engineering, IIT Delhi, Hauz Khas, New Delhi - 110016, 2012.

6. Ramamurthi B, Tandon PN. Text book of neuro surgery, $3^{\text {rd }}$ edition.

7. William Scoville. Lumbar disc surgery: technique of radical removal and early mobilization. Journal of Neurosurgery 1973;39(2):265-269. Online publication date: 1-Aug-1973.

8. Lee JJ, Patel R, Biermann JS, Dougherty PJ. The musculoskeletal effects of cigarette smoking. J Bone Joint Surg Am. 2013;95(9):850-9

9. Casper W. A new surgical procedure for lumbar disc herniation causing less tissue damage through a microsurgical approach.Adv Neurosurg 1977;4:74-80.

10. Moon HJ, Choi KH, Kim DH, et al. Effect of Lumbar Stabilization and Dynamic Lumbar Strengthening Exercises in Patients With Chronic Low Back Pain. Annals of Rehabilitation Medicine. 2013;37(1):110-117. doi: 1 0.5535/arm.2013.37. 1.1108 .

11. Ramani PS. The emergence of spinal surgery, micro lumbar discectomy, PLIF (posterior lumbar interbody fusion), reconstruction of traumatic cervical spine.

12. Young Baeg Kim. Clinical Applications of the Tubular Retractor on Spinal Disorders, Journal of Korean Neurosurgical Scoeity. 2007;42(4) 245-250. 